\title{
Status of the OLYMPUS Experiment
}

\author{
Ricardo Alarcon ${ }^{* \dagger}$ \\ Arizona State University \\ E-mail: ralarconeasu.edu

\section{Lauren Ice} \\ Arizona State University \\ E-mail: lauren.icedasu.edu
}

The OLYMPUS experiment will determine the multiple-photon exchange contribution to elastic lepton-proton scattering. The motivation for this experiment comes from an inconsistency in measurements of the proton electric-to-magnetic form factor ratio between the Rosenbluth separation and polarization techniques. It is expected that neglected multiple-photon exchange contributions to elastic lepton-proton scattering distort the extracted form factors and are the cause of this discrepancy. Determining the multiple-photon exchange contribution can be achieved by measuring the positron-proton to electron-proton elastic scattering cross section ratio. OLYMPUS was designed to measure this cross section ratio to better than $1 \%$ uncertainty for a wide range of four-momentum transfer and virtual photon polarization. The data for the ratio were taken in 2012 using $2.01 \mathrm{GeV}$ electron and positron beams incident on a hydrogen target. The data analysis is currently underway with preliminary results expected by November 2015 .

The 8th International Workshop on Chiral Dynamics, CD2015 ***

29 June 2015 - 03 July 2015

Pisa, Italy

* Speaker.

${ }^{\dagger}$ A footnote may follow. 


\section{Introduction}

Recent measurements of the proton electric-to-magnetic form factor ratio using polarization techniques show a disagreement with those made using the Rosenbluth Separation Technique. It is speculated that the cause for this disparity is a distortion in the measured form factors, stemming from a non-negligible multiple-photon exchange contribution to elastic lepton-proton scattering. Determining the multiple-photon exchange contribution can be achieved by comparing the positron-proton and electron-proton cross sections. Neglecting scattering amplitudes beyond the Born approximation, the positron-proton and electron-proton scattering cross sections are identical. The lowest order deviation from this is an interference term between the one-photon and twophoton exchange amplitudes that has opposite sign for positrons and electrons. OLYMPUS will use this to determine the multiple-photon exchange contribution by measuring the positron-proton to electron-proton elastic scattering cross section ratio to better than $1 \%$ uncertainty.

For the OLYMPUS experiment, electron and positron beams of $2.01 \mathrm{GeV}$ were scattered from an unpolarized hydrogen gas target and the scattering cross section ratio was measured using a wide acceptance spectrometer. The acceptance range covered by OLYMPUS, in momentum transfer squared, $\mathrm{Q}^{2}$, is from $0.25(\mathrm{GeV} / \mathrm{c})^{2}$ to $2.5(\mathrm{GeV} / \mathrm{c})^{2}$ and virtual photon polarization, $\varepsilon$, range is between 0.35 and 0.98 . This pushes our acceptance range wider than previous measurements and to large $\mathrm{Q}^{2}$ where the form factor discrepancy is largest. About $4 \mathrm{fb}^{-1}$ of data were taken during 2012 on the DORIS II ring at DESY Laboratory. Two other experiments, CLAS and VEPP-3 have also made this measurement and their results are published [1] and [2].

\section{Motivation}

\subsection{Electric and Magnetic Form Factors of the Proton}

Understanding the internal structure of nucleons is vital to our understanding of nuclear physics and provides crucial tests for hadronic physics, quantum chromodynamics, and nucleon-nucleon interactions. Lepton-proton scattering, in which the structure of the proton can be characterized by its influence on the scattered lepton, is essentail for investigating proton structure.

The influence of the proton electromagnetic field on the scattered lepton can be included in the cross section calculation by generalizing the lepton vertex to include structure functions, $\mathrm{F}_{1}$ and $\mathrm{F}_{2}$, which mathematically characterize the electromagnetic fields of the proton.

$$
\gamma^{\mu} \rightarrow \gamma^{\mu} \mathrm{F}_{1}\left(\mathrm{Q}^{2}\right)+i \sigma^{\mu v} \frac{\mathrm{q}_{v}}{2 \mathrm{~m}} \mathrm{~F}_{2}\left(\mathrm{Q}^{2}\right)
$$

$F_{1}$ and $F_{2}$ are the Pauli and Dirac form factors, and in the one photon exchange approximation, are only a function of $\mathrm{Q}^{2}$. The form factors can be rewritten as the electric and magnetic Sachs form factors, $G_{E}$ and $G_{M}$, which are more intuitively related to the charge and current distributions of the proton,

$$
\mathrm{G}_{\mathrm{E}}=\mathrm{F}_{1}-\tau \mathrm{F}_{2} \quad ; \quad \mathrm{G}_{\mathrm{M}}=\mathrm{F}_{1}+\mathrm{F}_{2}
$$

where $\tau=\frac{\mathrm{Q}^{2}}{2 \mathrm{M}}$ and $\mathrm{M}$ is the mass of the proton. The relation of $\mathrm{G}_{\mathrm{E}}$ and $\mathrm{G}_{\mathrm{M}}$ to the physical distributions of the charge and current are only valid in the Brett frame. With the form factors included, the first order approximation to the elastic scattering cross section can be written as, 


$$
\frac{\mathrm{d} \sigma}{\mathrm{d} \Omega}=\left(\frac{\mathrm{d} \sigma}{\mathrm{d} \Omega}\right)_{\text {Mott }}\left[\frac{\mathrm{G}_{\mathrm{E}}^{2}+\frac{\tau}{\varepsilon} \mathrm{G}_{\mathrm{M}}^{2}}{1+\tau}\right]
$$

where $\varepsilon=\left[1+2(1+\tau) \tan ^{2}\left(\theta_{e} / 2\right)\right]^{-1}$, is the virtual photon polarization, and the Mott scattering cross section is

$$
\left(\frac{\mathrm{d} \sigma}{\mathrm{d} \Omega}\right)_{\text {Mott }}=\frac{\alpha^{2}}{4 \mathrm{E}_{\mathrm{beam}}{ }^{2} \sin ^{4}\left(\theta_{e} / 2\right)} \frac{\mathrm{E}^{\prime} \cos ^{2} \theta_{e} / 2}{\mathrm{E}_{\mathrm{beam}}}
$$

where $\theta_{e}$ is the lepton scattering angle, $\alpha \approx 1 / 137$ is the fine structure constant, and $\mathrm{E}_{\text {beam }}$ and $\mathrm{E}^{\prime}$ are the beam and scattered lepton energies.

The proton form factors can be extracted from data using the Rosenbluth Separation Technique where the cross section is measured over a range of beam energies and electron scattering angles. From this, both $\mathrm{G}_{\mathrm{M}}$ and $\mathrm{G}_{\mathrm{E}}$ can be disentangled and extracted independently. When this measurement is carried out, [3-8], it is found that there is a rough proportionality between $\mathrm{G}_{\mathrm{E}}$ and $\mathrm{G}_{\mathrm{M}}$ with a dipole form factor,

$$
\mathrm{G}_{\mathrm{E}} \approx \frac{1}{\mu_{p}} \mathrm{G}_{\mathrm{M}} \approx \mathrm{G}_{\mathrm{D}} \approx\left(1+\frac{\mathrm{Q}^{2}}{0.71}\right)^{-2}
$$

where $\mu_{p}$ is the proton magnetic moment. At large $\mathrm{Q}^{2}$ it becomes increasingly difficult to extract $\mathrm{G}_{\mathrm{E}}$ because the $\frac{\tau}{\varepsilon} \mathrm{G}_{\mathrm{M}}^{2}$ term becomes large and dominates $\mathrm{G}_{\mathrm{E}}^{2}$ term.

The form factors of the proton can also be obtained using polarization observables. Two methods used are polarization transfer experiments and polarization asymmetry measurements. Polarization transfer experiments are conducted by scattering longitudinally polarized leptons on an unpolarized proton target and measuring the transverse, $\mathrm{P}_{\mathrm{x}}$, and longitudinal $\mathrm{P}_{\mathrm{Z}}$ components of the polarization of the recoiling proton. $P_{x}$ and $P_{z}$ are functions of $G_{E}$ and $G_{M}$ and from them the ratio of $G_{E}$ to $G_{M}$ can be extracted.

$$
\frac{\mathrm{G}_{\mathrm{E}}}{\mathrm{G}_{\mathrm{M}}}=-\frac{\mathrm{P}_{\mathrm{X}}}{\mathrm{P}_{\mathrm{Z}}} \frac{\left(\mathrm{E}_{\text {beam }}+\mathrm{E}^{\prime}\right)}{2 \mathrm{M}} \tan \left(\theta_{e} / 2\right)
$$

Polarization asymmetry experiments are conducted by scattering longitudinally polarized electrons off of a polarized proton target. The polarized cross section can be split into polarized and unpolarized parts,

$$
\sigma^{p o l}=\Sigma+h \Delta
$$

where $\Sigma$ is the unpolarized part given by equation (2.3), $h$ is the beam helicity and $\Delta$ is the polarized part of the cross section. My measuring the asymmetry in $\sigma^{\text {pol }}$ for the two beam helicities, an asymmetry, which is also a function of $\frac{\mathrm{G}_{\mathrm{E}}}{\mathrm{G}_{\mathrm{M}}}$ is as follows.

$$
A=\frac{-2 \sqrt{\tau(1+\tau)} \tan \left(\theta_{e} / 2\right) \frac{\mathrm{G}_{\mathrm{E}}}{\mathrm{G}_{\mathrm{M}}}}{\left(\frac{\mathrm{G}_{\mathrm{E}}}{\mathrm{G}_{\mathrm{M}}}\right)^{2}+\tau\left[1+2(1+\tau) \tan ^{2}\left(\theta_{e} / 2\right)\right]}
$$

From both the polarization transfer and asymmetry measurements the ratio of $G_{E}$ to $G_{M}$ can be extracted. Because only a ratio can be extracted, the issue of the scaling factors for $\mathrm{G}_{\mathrm{E}}$ are not present, and the ratio can be found to good precision even at high $\mathrm{Q}^{2}$. 
While experimental measurements using the Rosenbluth have been carried out for many decades, the polarization measurements only became viable since the advent of highly polarized lepton beams. As of today, many Rosenbluth method and polarization experiments have been conducted and the ratio $\frac{\mathrm{G}_{\mathrm{E}}}{\mathrm{G}_{\mathrm{M}}}$ found [9-21]. A sample of the data is shown in Figure 1.

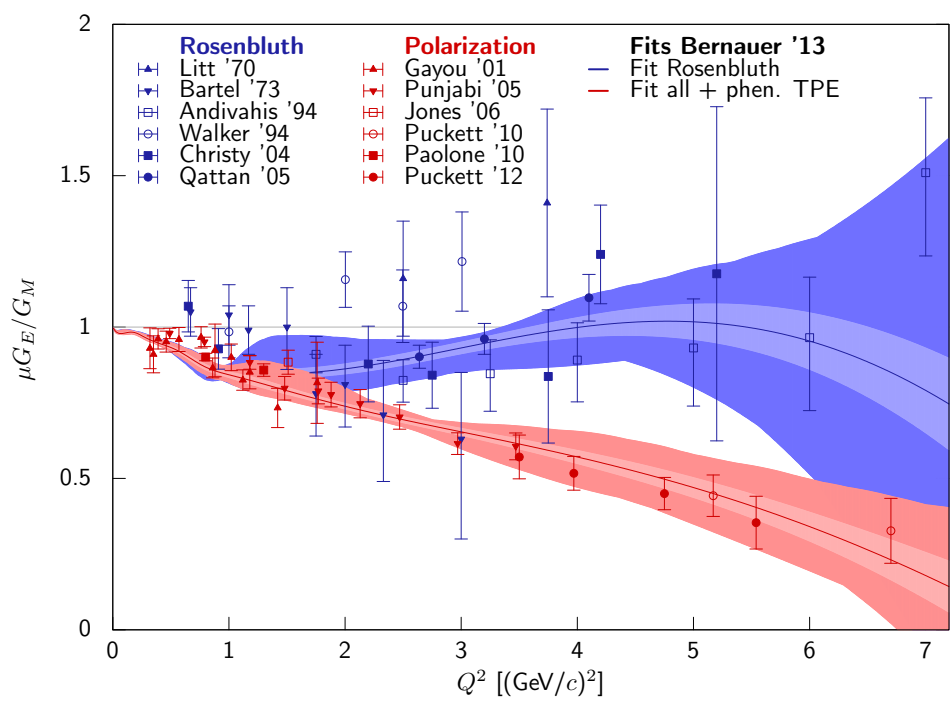

Figure 1: The $G_{E}$ to $G_{M}$ ratio as found with the Rosenbluth Separation Technique (blue) [3-8] and double polarization observables (red) [16-21]. Form factor fits to the world data also included [36]. The light bands are statistical uncertainty and the dark are model uncertainty.

From this, it is shown that with the Rosenbluth method, the form factor ratio is roughly constant over $\mathrm{Q}^{2}$, while a decrease in the ratio is apparent from polarization measurements. This discrepancy persists even into the ranges where both polarization and Rosenbluth techniques give precise results. It is speculated that this discrepancy is caused by higher-order amplitudes beyond the born approximation, distorting the extraction of the form factors, particularly those from unpolarized measurements. With polarization techniques, only form factor ratios are found minimizing the effect of higher-order amplitudes on the form factors. It is suggested that correcting for twophoton contributions in the Rosenbluth data can bring the form factor ratio measurements into agreement [22].

\subsection{Two-photon effects}

OLYMPUS will measure the multiple-photon exchange contribution by measuring the cross section ratio of positron-proton to electron-proton elastic scattering. Under the one-photon exchange assumption, the lepton-proton elastic cross section is insensitive to the charge of the lepton. When higher order scattering amplitudes are included, an interference term of order $\alpha^{3}$ between the one and the hard two-photon exchange amplitudes arises and is dependent on the charge sign 
of the lepton.

$$
\begin{gathered}
\sigma_{e^{-} p} \propto\left|\alpha M_{1 \gamma}-\alpha^{2} M_{2 \gamma}+\ldots\right|^{2}=\alpha^{2}\left|M_{1 \gamma}\right|^{2}-2 \alpha^{3} \operatorname{Re}\left\{M_{1 \gamma} M_{2 \gamma}\right\}+\ldots \\
\sigma_{e^{+} p} \propto\left|\alpha M_{1 \gamma}+\alpha^{2} M_{2 \gamma}+\ldots\right|^{2}=\alpha^{2}\left|M_{1 \gamma}\right|^{2}+2 \alpha^{3} \operatorname{Re}\left\{M_{1 \gamma} M_{2 \gamma}\right\}+\ldots \\
\frac{\sigma_{e^{-}}}{\sigma_{e^{+}}}=1+\frac{4 \operatorname{Re}\left\{M_{1 \gamma} M_{2 \gamma}\right\}}{\left|M_{1 \gamma}\right|^{2}}
\end{gathered}
$$

Therefore by precisely measuring the cross section ratio, the multiple photon exchange contribution can be observed through a deviation of the ratio from unity, proportional to the two-photon exchange amplitude.

Theoretical expectations for the results of $\frac{\sigma_{e^{+} p}}{\sigma_{e^{-}}}$vary significantly depending on the model of the proton structure [23-33]. Some of these predictions are shown in Figure 2.

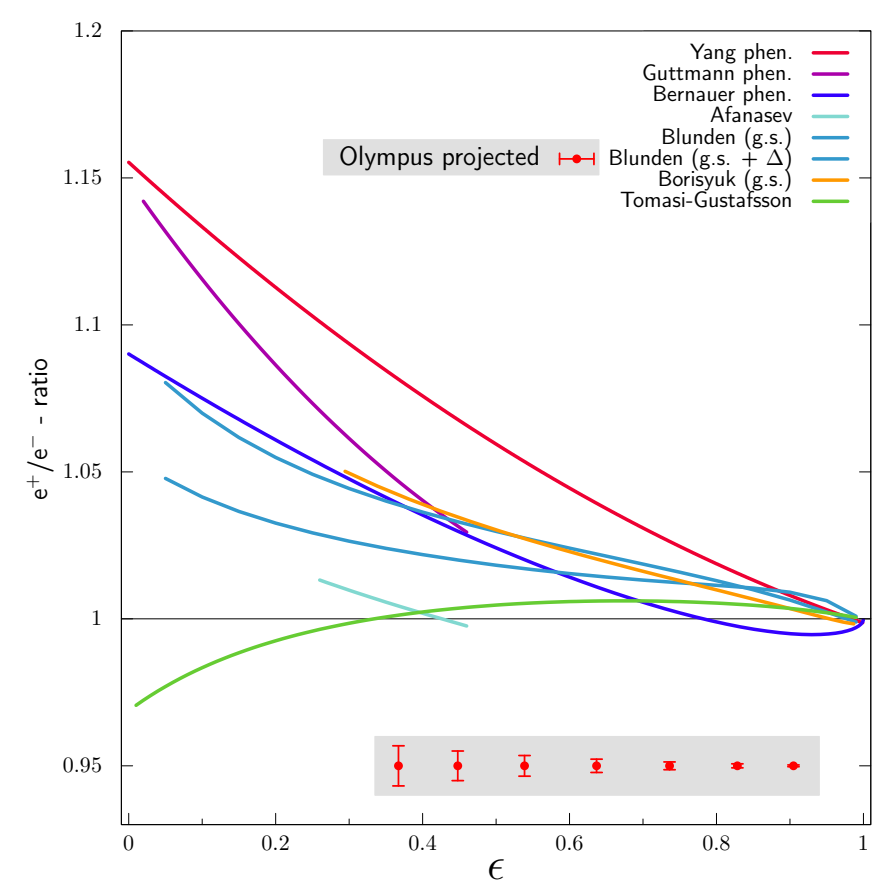

Figure 2: Some theoretical predictions for $\frac{\sigma_{e^{+} p}}{\sigma_{e^{-}}}$[23-32]. Several phenomological studies are also shown [34], [35], and [36]. The red points with gray background, are the expected $\varepsilon$ range and uncertainty for OLYMPUS.

\section{Experiment}

The OLYMPUS experiment was designed to measure the positron-proton to electron-proton elastic scattering cross section ratio to approximately $1 \%$ systematic and statistical uncertainty. A total of $4.45 \mathrm{fb}^{-1}$ of data were taken at the DORIS II storage ring at DESY Laboratory, in Hamburg Germany. Beams of $2.01 \mathrm{GeV}$ electrons and positrons were incident on an unpolarized hydrogen gas target. The cross section was measured using a large acceptance spectrometer. The approximate acceptance range OLYMPUS is $0.25 \leq \mathrm{Q}^{2} \leq 2.5(\mathrm{GeV} / \mathrm{c})^{2}$ and $0.35 \leq \varepsilon \leq 0.98$. 


\subsection{Experimental Setup}

The DORIS II storage ring at DESY Laboratory supplied 2.01 GeV electron and positron beams, incident on an unpolarized internal windowless hydrogen gas target. Hydrogen gas was pumped through an inlet at the center of the target cell and a multistage pumping system at both ends of the target restored the beam line vacuum. The target was cooled to below $70 \mathrm{~K}$ providing a target thickness of $3 \times 10^{15}$ atoms $/ \mathrm{cm}^{2}$. The target is described in detail in [38].

The detector systems were arranged around an eight-sector toroid magnet. A diagram of the detector is shown in Figure 3. A complete description of the detector can be found here [37].

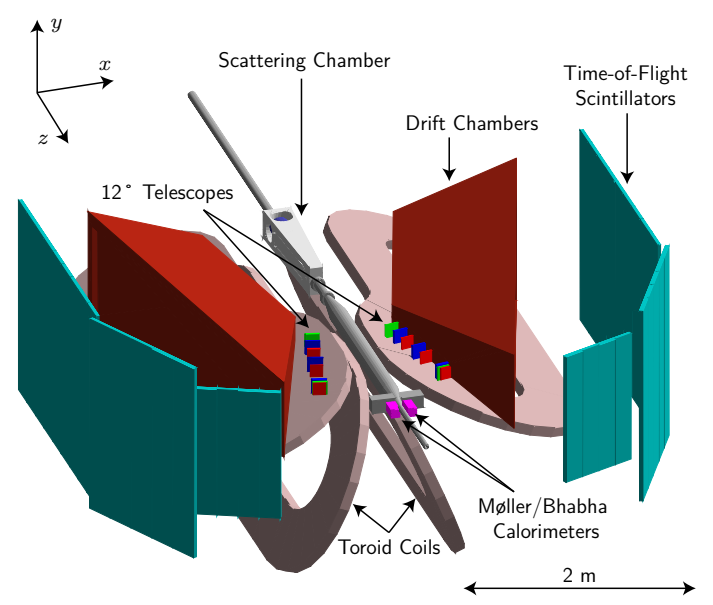

Figure 3: A solid model diagram of the OLYMPUS detector. For this diagram, the top magnet coils are removed so the detector components are visible.

The magnet provided track momentum and charge determination and swept the low energy Møller and Bhabha scattering forward, clear of the drift volume. In the horizontal planes of the magnet coils were six drift chambers, arranged in two single gas volumes, shown in red in Figure 3. These detectors provided information for track reconstruction and defined the acceptance range from approximately $20^{\circ}$ to $80^{\circ}$ scattering angle and $\pm 15^{\circ}$ azimuthal angle. Beyond the magnet, covering the full acceptance range of the drift chambers, were 36 time-of-flight (ToF) paddles, shown in aqua. In addition to measuring time of flight and giving an approximate energy calibration, they provided the trigger system. Last, at forward angles were two luminosity monitoring systems, the symmetric Møller and Bhabha calorimeters, and the $12^{\circ}$ telescopes.

\subsection{Luminosity Monitors}

Determining the $\frac{\sigma_{e^{+} p}}{\sigma_{e^{-}}}$to a systematic uncertainty close to $1 \%$ requires careful monitoring of the relative $e^{+}$and $e^{-}$luminosities. OLYMPUS had three systems to measure the luminosity. The first used run information such as beam current and target density (based on molecular flow simulations) to estimate the luminosity. This provided the luminosity with an approximate uncertainty of $3 \%$ relative and $15 \%$ absolute. The second luminosity monitors were two telescopes at approximately $12^{\circ}$ scattering angle. Each consisted of three gas electron multipliers (GEM) and three multiwire proportional chambers (MWPC). The $12^{\circ}$ monitors measured elastic lepton-proton scattering 
at low $\mathrm{Q}^{2}$ where the multiple-photon exchange contribution is expected to be small. Including theoretical uncertainties, the $12^{\circ}$ telescopes monitored the luminosity to an uncertainty better than $1 \%$ relative luminosity from approximately one hour of run time. The most precise luminosity monitoring system were two led-glass crystal calorimeters at $1.3^{\circ}$ scattering angle, designed to measure symmetric angle Møller and Bhabha scattering off of the target electrons. The calculations of these cross sections are known to high accuracy and the high rates provide a very precise and short time scale luminosity measurement.

\section{Analysis}

The analysis is currently in an advanced state, with a preliminary result expected at the American Physical Society Division of Nuclear Physics 2015 meeting. A detailed study of the other radiative corrections to elastic lepton-proton scattering, which can distort the cross section ratio measurement, is vital for determining the hard two photon exchange. Care is also taken to assure that the track reconstruction and analysis cuts do not bias the electron and positron beam data differently.

\subsection{Radiative Corrections}

In order to sift out the interference term between the one-photon and hard two-photon exchange amplitudes, other radiative corrections to lepton-proton scattering have to be corrected for in the measurement of the cross section ratio. The terms that are lepton-sign-odd, and therefore also cause the cross section ratio to deviate from one, are the soft two-photon exchange and Bremsstrahlung interference terms. The radiative corrections are implemented using a Monte Carlo simulation, allowing for detector efficiencies, acceptances, and analysis cuts to be folded in. The MC generates simulated lepton-proton scattering events weighted with the radiative cross section. This includes a full Bremsstrahlung calculation with no soft photon or peaking approximation. It also allows for several weights to be passed for each event, including the approximations of Maximon and Tjon [39] and Mo and Tsai [40].

Using Geant4, these events are propagated through the detector geometry. Each of the detector has a digitization simulation, which reproduces MC data in the same format as raw data and folds in detector acceptances and efficiencies. The digitized events are then run through the same reconstruction and analysis software as the data, properly accounting for reconstruction efficiencies and analysis cuts.

\subsection{Track Reconstruction and Analysis}

Track reconstruction in the OLYMPUS experiment is required to be accurate with little bias on lepton charge. The main tracking detectors are two drift chamber sectors placed in the two horizontal sections of the toroid. Each drift chamber sector consists of three chambers, made up of two superlayers. Each superlayer is a row of cells, each consisting of field wires and three sense wires. This provides 18 tracking planes, plus the time-of-flight scintillator array.

Several of the obstacles with track reconstruction encountered were large amounts of background noise, ambiguity track position through the cells, large inefficiencies, and complex time- 
to-distance functions for each cell. To help tackle these issues, several reconstruction techniques where implemented.

To help eliminate background, a MC pattern matching algorithm by M. Dell'orso and L. Ristori [41] was used for data clean-up and track finding. For this, a track pattern library was created with the MC simulation. The tracking then uses a tree search algorithm to match the data pattern with the track pattern library, and only signals matching a track pattern are reconstructed.

After pattern matching, a track reconstruction method, the Elastic Arms Algorithm (EAA) [43] and [42] recreates the tracks. The EAA method uses deformable template tracks (arms) defined only by the track kinematics, detector configuration and magnetic field. The arms are fit to the data through simulated deterministic annealing. This method helps to further eliminate noise and solves track position ambiguity in the drift chamber cells.

After elastic scattering selection on both real and simulated events, the final result for the ratio is given as

$$
\left(\frac{\sigma_{e^{+} p}}{\sigma_{e^{-}} p}\right)^{2}=\left(\frac{N_{e^{+} p}}{N_{e^{-}} p}\right)\left(\frac{L_{e^{-} p}}{L_{e^{+} p}}\right)\left(\frac{A_{e^{-} p}}{A_{e^{+} p}}\right)
$$

where, $N_{e^{ \pm} p}$ is the data rate ratio, $L_{e^{ \pm} p}$ is the relative luminosity measurements and $A_{e^{ \pm} p}$ is the acceptance function ratio defined from the Monte Carlo simulation rates.

\subsection{Yields}

After some preliminary analysis, plots showing the data and Monte Carlo comparison yields as a function of $\mathrm{Q}^{2}$ have been produced for a small subset of the data, The data has been blinded by removing events deteminted by two functions (one for electron beam and another for positron beam) in scattering angle. What the functions are, is unknown to most of the collaboration.

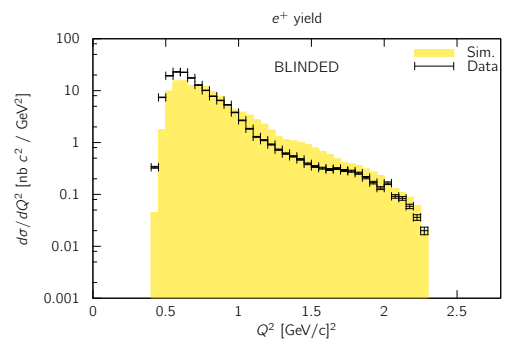

(a) Positron-proton scattering

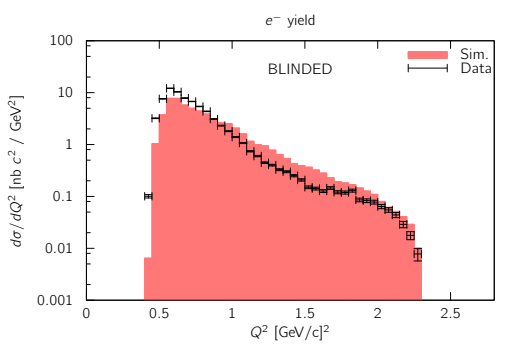

(b) Electron-proton scattering

Figure 4: Scattering yields (BLINDED) for approximately $2 \%$ of the data.

\section{Summary}

OLYMPUS will determine the multiple-photon exchange contribution to lepton-proton elastic scattering by measuring the positron-proton to electron-proton elastic scattering ratio to better than $1 \%$ uncertainty. The data for this experiment was taken in 2012 over a $\mathrm{Q}^{2}$ range from $0.25(\mathrm{GeV} / \mathrm{c})^{2}$ to $2.5(\mathrm{GeV} / \mathrm{c})^{2}$. The preliminary results will be released in the DNP 2015 meeting in Santa Fe, New Mexico. 


\section{References}

[1] D. Adikaram et al. (CLAS Collaboration) Phys. Rev. Lett. 114 (2015) 062003

[2] I. A. Rachek et al. Phys. Rev. Lett. 114 (2015) 062005

[3] J. Litt, G. Buschhorn, D. Coward, H. Destaebler, L. Mo, R. Taylor, B. Barish, S. Loken, J. Pine, J. Friedman, G. Hartmann, H. Kendall, Phys. Lett. B 31 (1970) 40-44

[4] W. Bartel et al. Nucl. Phys. B58 (1973)

[5] L. Andivahis et al. Phys. Rev. D50 (1994) 5491-5517

[6] R. C. Walker et al. Phys. Rev. D49 (1994) 5671-5689

[7] M. E. Christy et al. Phys. Rev. C70 (2004) 015206

[8] I. A. Qattan et al. Phys. Rev. Lett. 94 (2005) 142301

[9] B. D. Milbrath et al. Phys. Rev. Lett. 80 (1998) 452-455

[10] T. Pospischil et al. Eur. Phys. J. A12 (2001) 125-127

[11] B. Hu et al. Phys. Rev. C73 (2006) 064004

[12] G. MacLachlan et al. Nucl. Phys. A764 (2006) 261-272

[13] C. B. Crawford et al. Phys. Rev. Lett. 98 (2007) 052301

[14] G. Ron et al. Phys. Rev. C84 (2011) 055204

[15] X. Zhan et al. Phys. Lett. B705 (2011) 59-64

[16] O. Gayou et al. Phys. Rev. C64 (2001) 038202

[17] V. Punjabi et al. Phys. Rev. C71 (2005) 055202

[18] M. K. Jones et al. Phys. Rev. C 74 (2006) 035201

[19] A. J. R. Puckett et al. Phys. Rev. Lett. 104 (2010) 242301

[20] M. Paolone, S. P. Malace, S. Strauch, I. Albayrak, J. Arrington, et al. Phys. Rev. Lett. 105 (2010) 072001

[21] A. J. R. Puckett et al. Phys. Rev. C85 (2012) 045203

[22] J. Arrington, W. Melnitchouk, J. A. Tjon, Phys. Rev. C76 (2007) 035205

[23] P. A. Guichon, M. Vanderhaeghen, Phys. Rev. Lett. 91 (2003) 142303

[24] P. Blunden, W. Melnitchouk, J. Tjon, Phys. Rev. Lett. 91 (2003) 142304

[25] Y. C. Chen, A. Afanasev, S. J. Brodsky, C. E. Carslon, M. Vanderhaeghen, Phys. Rev. Lett. 93 (2004) 122301

[26] A. V. Afanasev, S. J. Brodsky, C. E. Carlson, Y. C. Chen, M. Vanderhaeghen, Phys. Rev. D 72 (2005) 013008

[27] P. G. Blunden, W. Melnitchouk, J. A. Tjon, Phys. Rev. C 72 (2005) 034612

[28] S. Kondratyuk, P. G. Blunden, W. Melnitchouk, J. A. Tjon, Phys. Rev. Lett. 95 (2005) 172503

[29] D. Borisyuk, A. Kobushkin, Phys, Rev. C74 (2006) 065203

[30] E. Tomasi-Gustafsson, M. Osipenko, E. Kuraev, Y. Bystritsky, Phys. Atom. Nucl. 76 (2013) 937-946 
[31] E. Tomasi-Gustafsson, G. Gakh, Phys. Rev. C72 (2005) 015209

[32] Y. M. Bystritsky, E. A. Kuraev, E. Tomasi-Gustafsson, Phys. Rev. C75 (2007) 015207

[33] M. Gorchtein, Phys. Lett. B644 (2007) 322-330

[34] Y. C. Chen, C. W. Kao, S. N. Yang, Phys. Lett. B652 (2007) 269-274

[35] J. Guttmann, N. Kivel, N. Mezaine, M. Vanderhaeghen, Eur. Phys. J. A47 (2011) 1-5

[36] J. C. Bernauer et al. Phys. Rev. C (2013)

[37] R. Milner et al. (OLYMPUS Collaboration) Nucl. Inst. Meth. A 741 (2014) 1-17

[38] J.C. Bernauer, V. Carassiti, G. Ciullo, B.S. Henderson, E. Ihloff, J. Kelsey, P. Lenisa, R. Milner, A. Schmidt, M. Statera, Nucl. Inst. Meth. A755 (2014) 20-27

[39] L.C. Maximon, J.A. Tjon, Phys. Rev. C62 (2000) 054320

[40] L.W. Mo and Y.S. Tsai, Rev. Mod. Phys. 41, 205 (1969)

[41] M. Dell'Orso, L. Ristori, Nucl. Inst. Meth. A 287 (1990) 436-438

[42] M. Ohlsson, Comp. Phys. Comm. 77 (1993) 19-32

[43] M. Ohlsson, C. Peterson, A.L. Yuille, Comp. Phys. Comm. 71(1992) 77-98 